\title{
CpG site degeneration triggered by the loss of functional constraint created a highly polymorphic macaque drug-metabolizing gene, CYP1A2
}

Yasuhiro Uno ${ }^{1}$ and Naoki Osada $2,3^{*}$

\begin{abstract}
Background: Elucidating the pattern of evolutionary changes in drug-metabolizing genes is an important subject not only for evolutionary but for biomedical research. We investigated the pattern of divergence and polymorphisms of macaque CYP1A1 and CYP1A2 genes, which are major drug-metabolizing genes in humans. In humans, CYP1A2 is specifically expressed in livers while CYP1A1 has a wider gene expression pattern in extrahepatic tissues. In contrast, macaque CYP1A2 is expressed at a much lower level than CYP1A1 in livers. Interestingly, a previous study has shown that Macaca fascicularis CYP1A2 harbored unusually high genetic diversity within species. Genomic regions showing high genetic diversity within species is occasionally interpreted as a result of balancing selection, where natural selection maintains highly diverged alleles with different functions. Nevertheless many other forces could create such signatures.

Results: We found that the CYP1A1/2 gene copy number and orientation has been highly conserved among mammalian genomes. The signature of gene conversion between CYP1A1 and CYP1A2 was detected, but the last gene conversion event in the simian primate lineage occurred before the Catarrhini-Platyrrhini divergence. The high genetic diversity of macaque CYP1A2 therefore cannot be explained by gene conversion between CYP1A1 and CYP1A2. By surveying CYP1A2 polymorphisms in total $91 \mathrm{M}$. fascicularis and M. mulatta, we found several null alleles segregating in these species, indicating functional constraint on CYP1A2 in macaques may have weakened after the divergence between humans and macaques. We propose that the high genetic diversity in macaque CYP1A2 is partly due to the degeneration of $\mathrm{CPG}$ sites, which had been maintained at a high level by purifying selection, and the rapid degeneration process was initiated by the loss of functional constraint on macaque CYPIA2.

Conclusions: Our findings show that the highly polymorphic CYP1A2 gene in macaques has not been created by balancing selection but by the burst of $\mathrm{CpG}$ site degeneration after loss of functional constraint. Because the functional importance of CYP1A1/2 genes is different between humans and macaques, we have to be cautious in extrapolating a drug-testing data using substrates metabolized by CYP1A genes from macaques to humans, despite of their somewhat overlapping substrate specificity.
\end{abstract}

\section{Background}

Cytochrome P450 oxidase (CYP) proteins oxidize a wide variety of substrates. They could metabolize not only many endogenous substrates such as steroid hormones, but also exogenous toxins for excretion [1]. In

\footnotetext{
* Correspondence: nosada@lab.nig.ac.jp

${ }^{2}$ Department of Population Genetics, National Institute of Genetics, 1111

Yata, Mishima, Shizuoka 411-8540, Japan

Full list of author information is available at the end of the article
}

biomedical research, CYP are widely recognized as drug-metabolizing enzymes. Probably, normal function of drug-metabolizing CYPs in natural habitats would be detoxication of xenophobic chemicals taken from foods, especially from plants [2].

Humans have about 60 CYP genes in the genome [3]. Among them, CYP1A gene subfamily is one of the most major $C Y P$ subfamilies in humans. The human genome contains two CYP1A genes on chromosome 15, CYP1A1
C Biomed Central

(c) 2011 Uno and Osada; licensee BioMed Central Ltd. This is an Open Access article distributed under the terms of the Creative Commons Attribution License (http://creativecommons.org/licenses/by/2.0), which permits unrestricted use, distribution, and reproduction in any medium, provided the original work is properly cited. 
and CYP1A2, which are tandemly aligned with $23-\mathrm{kb}$ interval and head-to-head orientation [4]. The amino acid sequence identity between the paralogs is $>80 \%$ and their protein structures are supposed to be very similar, partly accounting for overlapping substrate selectivity of these enzymes [5]. Human CYP1A2 is constitutively expressed in livers and contributes to the hepatic metabolism of many important chemical compounds such as caffeine [6]. In contrast, the tissue distribution of human CYP1A1 gene expression is very broad and its expression is strongly induced by exogenous compounds, indicating CYP1A2 rather than CYP1A1 is a major hepatic $C Y P 1 A$ gene in humans. Polymorphisms in human $C Y P 1 A$ genes have been intensively studied for medical benefits (e.g., [7]), while a few studies from an evolutionary standpoint have been conducted for human $C Y P 1 A$ genes $[8,9]$. For example, Jorge-Nebert et al. surveyed the pattern of single nucleotide polymorphisms (SNPs) in several human populations at the CYP1A1-CYP1A2 locus and found the signature of selective sweep around the CYP1A1 untranslated region [9]. However, the role of the selective sweep for human CYP1A1 evolution remains unclear.

Because macaque monkeys are widely used for testing drug toxicity in preclinical trials, the genetic similarity of CYP genes between humans and macaques is important not only for evolutionary research but also for biomedical applications [10]. Unexpected phenotypic difference between divergent species is one the biggest concerns in drug development, e.g., [11]. A previous study has suggested that drug metabolism by CYP1A1/2 was not perfectly conserved among humans, macaques, and marmosets [12]. Elucidating the cause of phenotypic differences among primates at a genetic level is an important task.

Our previous sequence analysis showed that the region encompassing the first intron and exon 2 of macaque CYP1A2 had the highest genetic diversities among randomly selected 54 unlinked autosomal loci [13]. Because allelic variation of CYP1A2 proteins may increase a repertory of toxic substrates metabolized by CYP1A2, it is reasonable to imagine that the high genetic diversity in macaque CYP1A2 has been maintained by balancing selection, where natural selection maintains highly diverged alleles having different functions $[14,15]$. However, other studies also have shown that the gene expression pattern of CYP1A2 in macaques was quite different from that in humans. The level of gene expression of macaque CYP1A2 is very low in all Macaca fascicularis (cynomolgus macaques) livers so far examined $[16,17]$. The expression level of CYP1A1 is instead much more abundant in macaque livers. Interestingly, the pattern of CYP1A1/2 protein expression appears similar between humans and marmosets [12], indicating a major hepatic CYP1A gene was switched at some time after the divergence of the human and macaque lineages [17]. The result casts doubt that the balancing selection hypothesis accounts for high CYP1A2 diversity in macaques. However, if macaque $C Y P 1 A 2$ lost its function, the evolutionary history of CYP1A2 should resemble many other neutral loci.

In order to answer the question why macaque CYP1A2 has unusually high genetic diversity, we first investigated the long-term evolution pattern of $C Y P 1 A$ subfamily. Gene conversion between paralogs was examined with particular attention, because gene conversion between paralogs may create an unusual pattern of polymorphisms in paralogous genes. We found that the last gene conversion event between CYP1A1 and CYP1A2 in the lineages to Catarrhini primates occurred after the divergence of primates and rodents, and before the divergence of Catarrhini and Platyrrhini primates. We further sequenced $C Y P 1 A 1 / 2$ coding regions of $63 \mathrm{M}$. fascicularis and $28 \mathrm{M}$. mulatta individuals, and found several deletions and single nucleotide polymorphisms in macaque CYP1A2 with relatively high frequencies, which cause premature stop codons. We propose that macaque CYP1A2 is in the process of pseudogenization, and rapid degeneration of $\mathrm{CpG}$ sites is creating a high genetic diversity.

\section{Results}

Phylogenetic analysis of mammalian CYP1A subfamily

We first analyzed the general pattern of molecular evolution in the mammalian CYP1A subfamily. The phylogenetic tree of $C Y P 1 A 1 / 2$ genes was constructed using the cDNA sequences registered in the public database (Figure 1). Although two independent gene duplications were assigned on the phylogenetic tree, Goldstone and Stegeman [18] have suggested that intense inter-locus

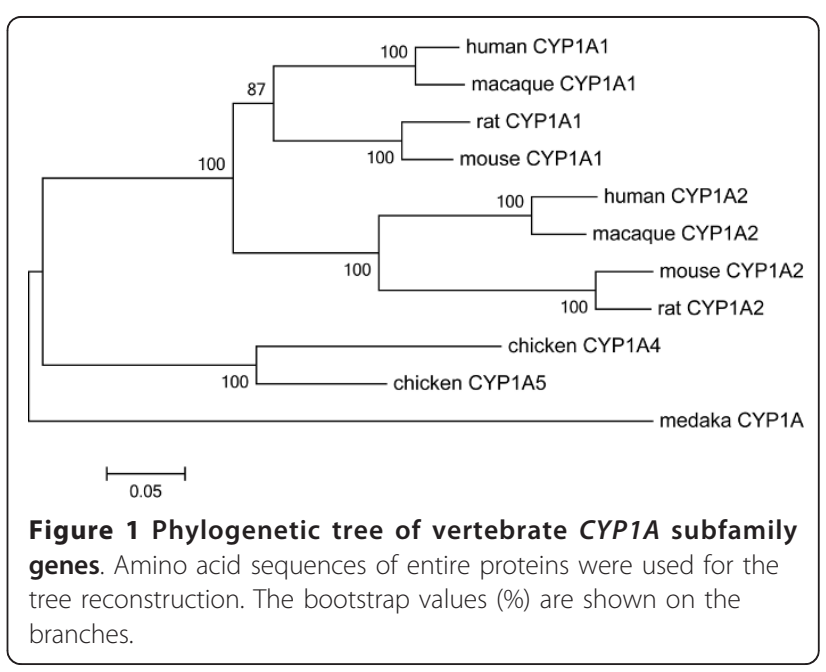


gene conversion occurred in the lineage of chickens; i.e., mammalian CYP1A1/2 genes and chicken $C Y P 1 A 5 / 4$ genes are orthologous, respectively. We further searched syntenic regions to the human CYP1A1-CYP1A2 locus from the draft genome sequences of several vertebrate genomes. We found that teleost fish (medaka) and amphibian (xenopus) genomes contained only one CYP1A gene, while chicken, opossum, and other placental mammalian genomes (human, orangutan, macaque, mouse, rat, rabbit, guinea pig, cow, horse, dog, and elephant) encompassed two genes in a head-to-head orientation. In addition, a previous study has shown that all fish CYP1A genes formed a monophyletic cluster [5]. Therefore, gene duplication of $C Y P 1 A 1 / 2$ was supposed to occur at some time between the Amphibia-Amniota divergence and Mammalia-Sauropsida divergence, around 300-350 Mya [19,20], and the number of genes has been stable since the gene duplication.

We also investigated whether macaques carry additional copy of $C Y P 1 A$ genes other than $C Y P 1 A 1 / 2$ in the genome. The latest draft genome sequence of $M$. mulatta (rheMac2) did not contain any other sequence similar to CYP1A. Furthermore, a previous search for genes expressed in $M$. fascicularis livers did not identify any additional macaque $C Y P 1 A$ paralogs [21], suggesting that $C Y P 1 A 1$ and $C Y P 1 A 2$ are the only CYP1A genes in macaque genomes.

\section{Gene conversion between CYP1A1 and CYP1A2}

The pattern of paralogous divergence between CYP1A1 and $C Y P 1 A 2$ genes was further investigated. In order to grasp an overall picture, we first focused on only human and mouse genes. Amino acid differences ( $p$-distance) between human and mouse orthologs and between paralogs for each coding exon are summarized in Table 1 . Considering that the gene duplication of CYP1A1/2 occurred more than 300 Mya and the divergence between primates and rodents occurred around 80-120 Mya, paralogous genes should have much more diverged than human-mouse orthologs. Nevertheless, in exon 2 and 5 , the average level of protein divergence between paralogous genes was smaller than that between humanmouse orthologs. The pattern suggests that gene conversion between CYP1A1 and CYP1A2 may have

Table 1 Amino acid $p$-distance between human-mouse CYP1A1/2 quartets

\begin{tabular}{lllllll}
\hline & Exon2 & Exon3 & Exon4 & Exon5 & Exon6 & Exon7 \\
\hline hCYP1A1-mCYP1A1 & 0.222 & 0.167 & 0.034 & 0.103 & 0.107 & 0.266 \\
hCYP1A2-mCYP1A2 & 0.289 & 0.263 & 0.310 & 0.179 & 0.250 & 0.253 \\
hCYP1A1-hCYP1A2 & 0.216 & 0.569 & 0.172 & 0.128 & 0.179 & 0.309 \\
mCYP1A1-mCYP1A2 & 0.215 & 0.655 & 0.241 & 0.051 & 0.214 & 0.372 \\
\hline
\end{tabular}

* $h$ and $m$ represent human and mouse genes, respectively. homogenized the paralogous gene sequences after the divergence of primates and rodents.

In order to detect gene conversion between $C Y P 1 A 1$ and $C Y P 1 A 2$, we analyzed the human-mouse quartet gene set using a maximum likelihood framework. Figure 2A shows

A
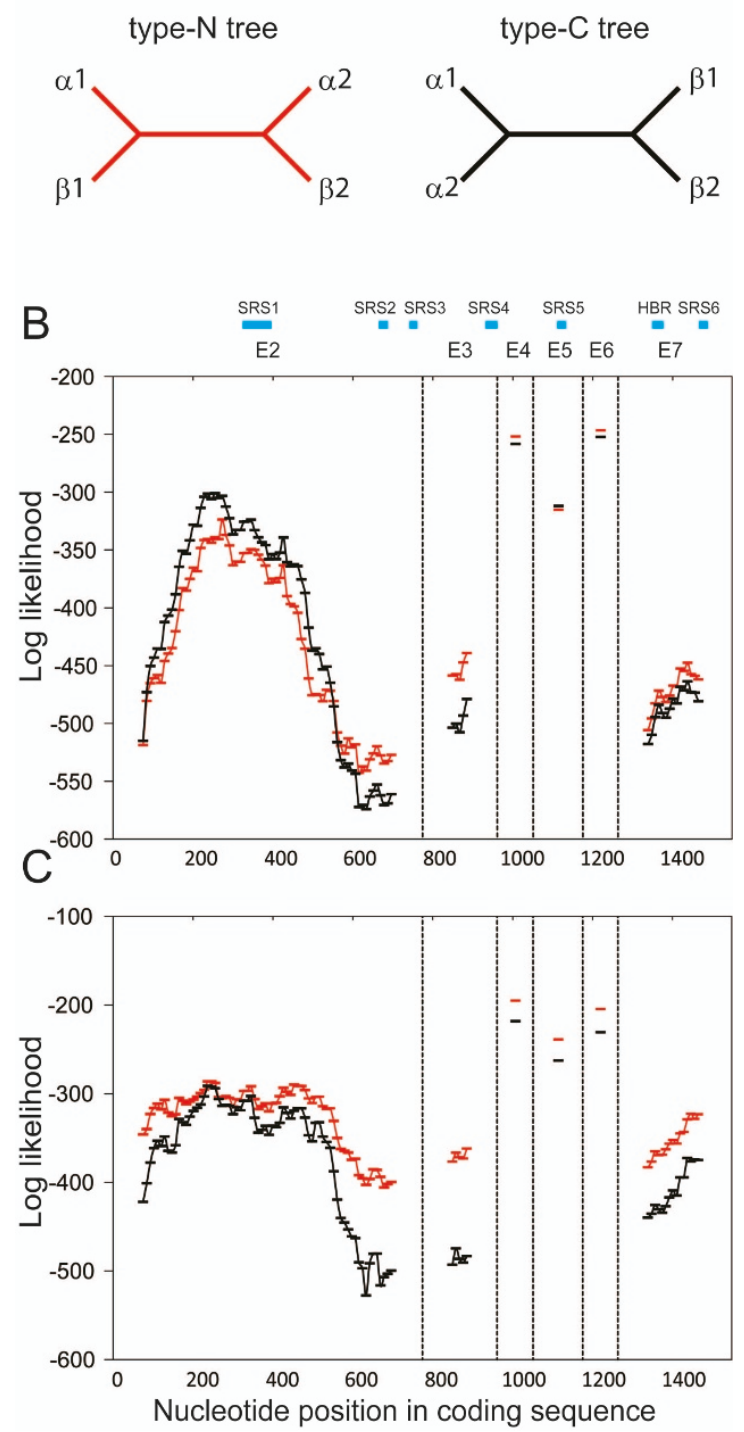

Figure 2 Test of region-specific gene conversion. Gene duplication occurred before the divergence of two species ( $\alpha$ and $\beta$ ) and each species has two copies of genes (1 and 2). A) When gene conversion occurred after the divergence of two species, the tree topology would become the type- $C$ tree (right); otherwise the tree would become the type-N tree (left). B) The log-likelihood values of the type- $\mathrm{N}$ (red lines) and type- $\mathrm{C}$ trees (black lines) in the human-mouse quartet genes. Substrate recognition sites (SRS) and heme-binding regions (HBR) are shown as the blue squares. The vertical dashed lines represent boundaries between exons. C) The log-likelihood values in the human-marmoset quartet genes. 
the expected patterns of paralogous gene divergence in two species when the gene duplication preceded the split of species. If gene conversion between paralogs does not occur, a type- $\mathrm{N}$ tree in Figure 2A would be observed. On the other hand, if gene conversion homogenized the two paralogous sequences, the phylogenetic pattern among the quartet genes would become a type- $\mathrm{C}$ tree in Figure 2A. Since the two types of trees are unrooted trees, the topology of trees with gene conversion only in one species would become the same as the type- $\mathrm{C}$ tree. In order to examine the regional heterogeneity of divergence pattern, we binned exon 2, 3, and 7 into 150-bp-length windows with 9-bp (3 codons) sliding steps; exon 4, 5, and 6 were not subdivided because they were shorter than $150 \mathrm{bp}$. For each window, likelihood values of the type- $\mathrm{N}$ and type- $\mathrm{C}$ trees were maximized using PAML [22]. If gene conversion occurred after the divergence of primates and rodents, those regions would have a higher probability of the type- $\mathrm{C}$ tree than that of the type- $\mathrm{N}$ tree. Between humans and mice, the first $500 \mathrm{bp}$ of exon 2 and entire exon 5 had higher likelihood values of the type- $\mathrm{C}$ tree than that of the type-N tree (Figure 2B), indicating gene conversion had homogenized these regions after the divergence of primates and rodents. The highest log-likelihood ratio was 39.3 in the window at $237 \mathrm{bp}$, which corresponded to $P<10^{-22}$. The regions under gene conversion encompassed substrate recognition site (SRS) 1 and 5. We repeated the analysis using a human-marmoset quartet set. Interestingly, the high-probability regions of the type$\mathrm{C}$ tree were not observed in the human-marmoset quartets (Figure 2C), suggesting that gene conversion did not occur in the Catarrhini lineage after the divergence of Catarrhini and Platyrrhini.

\section{Genetic diversity of CYP1 genes in M. fascicularis}

Since gene conversion could not explain the high genetic diversity of macaque CYP1A2, detailed analyses on macaque $C Y P 1 A 1 / 2$ polymorphisms were conducted. We determined complete coding sequences of CYP1A1 and CYP1A2 genes of 10 and $7 \mathrm{M}$. fascicularis individuals, respectively, by sequencing PCR amplicons of genomic DNA. Basic population genetic statistics such as nucleotide diversity $(\pi)$, number of segregating sites $(S)$, and Tajima's $D$ are summarized in Table 2. CYP1A1 showed less genetic diversity than CYP1A2 both at

Table 2 Genetic diversity of M.fascicularis CYP1A1/2 genes

\begin{tabular}{lllllll}
\hline & $\boldsymbol{N}$ & $\boldsymbol{S}$ & $\boldsymbol{\pi}$ & $\boldsymbol{\pi}_{\mathbf{S}}$ & $\boldsymbol{\pi}_{\mathbf{A}}$ & $\boldsymbol{D}$ \\
\hline CYP1A1 & 20 & 7 & 0.00072 & 0.00219 & 0.00026 & -1.446 \\
CYP1A2 & 14 & 31 & 0.00462 & 0.00740 & 0.00375 & -1.139
\end{tabular}

${ }^{*} N$, number of sampled chromosomes; $S$, number of segregating sites; $\pi$, nucleotide diversity, at synonymous sites $\left(\pi_{\mathrm{S}}\right)$ and nonsynonymous sites $\left(\pi_{\mathrm{A}}\right)$; $D$, Tajima's $D$ statistics synonymous and nonsynonymous sites. Both genes showed similar level of negative Tajima's $D$ values as those in other loci studied in the previous study (average was -0.68 with 0.64 standard deviation in 27 randomly selected genes [13]). Although negative $D$ statistics might have been caused by selective force around the locus such as selective sweep, the prevalence of negative $D$ statistics in the genome suggested the past population expansion of M. fascicularis and M. mulatta.

In those samples, four null mutations were segregating in M. fascicularis CYP1A2. If the function of CYP1A2 were completely lost before the most recent common ancestor (MRCA) of macaques, mutations would be randomly segregating at nonsynonymous and synonymous sites and the ratio of per-site nucleotide diversity at nonsynonymous site to synonymous sites $\left(\pi_{\mathrm{A}} / \pi_{\mathrm{S}}\right)$ would be close to 1 . The ratio of nonsynonymous nucleotide diversity $\left(\pi_{\mathrm{A}}\right)$ to synonymous nucleotide diversity $\left(\pi_{\mathrm{S}}\right)$ in CYP1A2 was 0.51 , much smaller than 1 , although larger than the ratio in CYP1A1 $\left(\pi_{\mathrm{A}} / \pi_{\mathrm{S}}=0.12\right)$. The ratio smaller than 1 indicates that the CYP1A2 gene in macaques lost its function after the occurrence of MRCA, or the function of $C Y P 1 A 2$ is partly retained: i.e., null alleles have been weakly selected against in macaque CYP1A2. When we analyzed only individuals that have both CYP1A1 and CYP1A2 sequences (12 chromosomes), the estimated values of $\pi$ were similar to the values with all samples (0.00075 and 0.00442 in $M$. fascicularis CYP1A1 and CYP1A2, respectively).

In order to investigate whether the different pattern of genetic diversity between $C Y P 1 A 1$ and $C Y P 1 A 2$ genes are simply due to difference in functional constraint and/or mutation rates, HKA tests were conducted using human orthologous genes as outgroup genes [23]. $M$. fascicularis individuals having both CYP1A1 and $C Y P 1 A 2$ sequences were analyzed and the test was significant $(P=0.0415)$, indicating macaque $C Y P 1 A 2$ has relatively high polymorphism given the level of divergence. The marginal significance in $M$. fascicularis was improved $(P=0.0224)$ when an additional eight $M$. mulatta chromosomes were analyzed together.

\section{Resequencing of a large number of $M$. fascicularis and $M$. mulatta CYP1A2}

To elucidate the mechanisms for creating polymorphisms in macaque CYP1A2, we sequenced the first 1166 bp of the coding sequences, which spanned from exon 2 to exon 4, of additional $63 \mathrm{M}$. fascicularis and $28 \mathrm{M}$. mulatta samples. We identified five null mutations in those samples (Table 3). Among those individuals, 38 and 7 macaques carried null alleles in the coding regions as heterozygotes and homozygotes, respectively. For the null mutations of high frequency at site 1066, Hardy-Weinberg equilibrium was examined. In both $M$. 
Table 3 Allele frequency of null alleles in macaque CYP1A2

\begin{tabular}{llll}
\hline $\begin{array}{l}\text { Position in coding } \\
\text { sequence }\end{array}$ & Type & $\begin{array}{l}\text { M. } \\
\text { fascicularis }\end{array}$ & $\begin{array}{l}\text { M. } \\
\text { mulatta }\end{array}$ \\
\hline 138 & G to A & $0 / 126$ & $5 / 51$ \\
286 & 1 bp & $4 / 122$ & $0 / 56$ \\
& deletion & & \\
1066 & C to T & $8 / 118$ & $22 / 34$ \\
1090 & C to T & $2 / 124$ & $0 / 56$ \\
1103 & T to A & $11 / 115$ & $0 / 56$ \\
\hline
\end{tabular}

*The number of null alleles is shown in the left of slash (/).

fascicularis and $M$. mulatta, the deviation from the equilibrium was not detected $(P=0.874$ and $P=0.753$, respectively; chi-square test). The prevalence of null alleles and homozygous individuals supports that $C Y P 1 A 2$ is a dispensable gene in macaques and the effect of purifying selection on macaque CYP1A2 is considerably weak. Among 65 SNPs in this region, 36 were $M$. fascicularis specific, 16 were M. mulatta specific, and 13 were shared between species. No fixed difference between species was discovered.

Interestingly, 32 of 65 SNPs were at $\mathrm{CpG}$ dinucleotide sites. It is well known that methylated dinucleotide CG tends to mutate to CA and TG in mammalian genomes, probably at $>10$ times faster than the background mutation rate $[24,25]$. In agreement with this prediction, 30 of 32 mutations at $\mathrm{CpG}$ sites were mutations from CG to CA or from CG to TG, assuming human CYP1A2 had ancestral alleles. Among those mutations, 20,9 , and 1 were synonymous, nonsynonymous, and null mutations, respectively. We counted the number of CpG dinucleotides in the reference $M$. fascicularis CYP1A1/2 cDNA sequences (CYP1A1, D17575; $C Y P 1 A 2, \mathrm{D} 86474)$. Macaque $C Y P 1 A 1$ and $C Y P 1 A 2$ had 41 and $63 \mathrm{CpG}$ dinucleotides in the protein coding regions, respectively; the macaque $C Y P 1 A 2$ contained CpG sites about $8 \%$ of the coding sequence. Among all annotated genes in the $M$. mulatta genome sequence (RefSeq genes, [26]), the fraction of CpG sites in CYP1A2 was ranked in the upper $8.3 \%$.

Many of CpG degenerations (GC to TG and GC to GA) within a gene are nonsynonymous changes and those $\mathrm{CpG}$ sites should be protected by purifying selection from degeneration if the gene is important for survival. In mammalian genomes, GC content at synonymous sites are also generally higher than that at intergenic non-coding sites [27]. We therefore hypothesized that the loss-of-function of macaque CYP1A2 (either completely or partially) may have initiated the degenerative substitutions at $\mathrm{CpG}$ sites in the gene. In order to test this hypothesis, we reanalyzed the polymorphism data of $24 \mathrm{M}$. fascicularis individuals in 27 genic (exons and adjacent non-coding sequences) and
27 intergenic regions (at least $100 \mathrm{~kb}$ away from any coding regions), which were randomly distributed on the autosomes and about $40 \mathrm{~kb}$ length in total [13]. As expected, the genic regions harbored more $\mathrm{CpG}$ sites per nucleotide than intergenic regions $(P=0.0047$, Wilcoxon test). In Figure 3, nucleotide diversity and fraction of CpG sites are plotted for intergenic and genic regions. Statistically significant positive correlation (Spearman's $\rho=0.45, P=0.020$ ) was observed in the intergenic regions, while no significant correlation was observed in the genic regions (Spearman's $\rho=-0.019, P$ $=0.93)$. The results indicate that the level of polymorphisms in intergenic regions is strongly affected by the number of $\mathrm{CpG}$ sites in those regions, whereas the functionality of genic regions prevents $\mathrm{CpG}$ site degeneration.

\section{Discussion}

\section{Natural selection on drug-metabolizing genes}

The process of drug metabolism has been under intense studies for biomedical benefits. Because humans are only natural organisms utilizing so-called "drugs", the impact of natural selection on drug metabolizing genes still remains unclear, except for some cases of insect herbivores specialized to their host plants $[28,29]$. Considering that many plant secondary metabolites are oxidized by CYP proteins and endogenous substrates controlling physiology such as hormones are also metabolized by CYP proteins, it is straightforward to access the effect of natural selection on the CYP protein diversity. For example, Thompson et al. proposed that the natural variation in human CYP3A5 is related to the retention of salt in a body and under natural selection

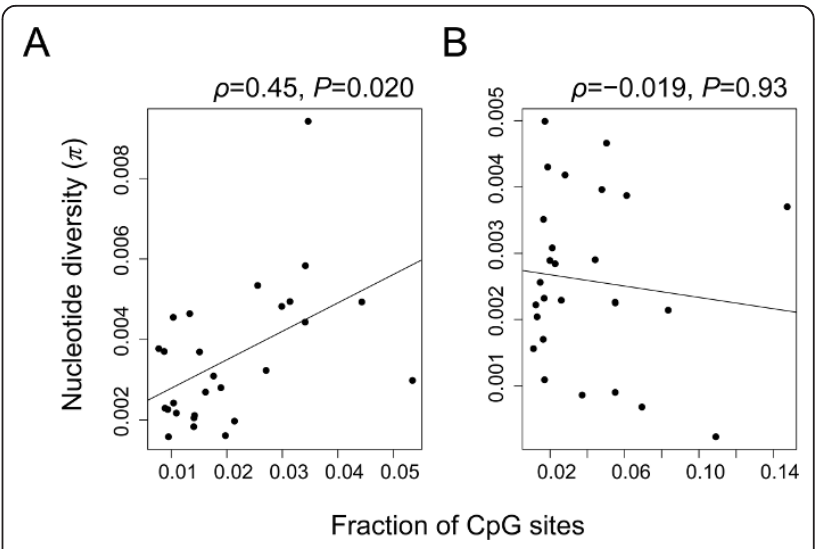

Figure 3 Fraction of $\mathrm{CpG}$ sites and nucleotide diversity in $M$. fascicularis. The data is obtained from Osada et al. [13]. Liner regression lines are represented as the solid lines. Note that the correlation test was performed using a non-parametric test (Spearman's rank correlation test). A) Intergenic regions that are away from at least $100 \mathrm{~kb}$ from any annotated genes. B) Genic regions containing at least one exon. 
according to latitudes [30]. A scan of recent selective sweeps in the human genome also detected a significant excess of CYP genes among those regions [31]. In macaques, Osada et al. found that CYP3A5 gene was highly differentiated between $M$. fascicularis and M. mulatta compared with other genes, suggesting the action of natural selection on the gene for local adaptation [13]. In the same study, they also found that CYP1A2 contained unusually high genetic diversity within the macaque species. Although the previous studies have revealed a weak hepatic expression of macaque CYP1A2, the weak expression does not necessary imply that macaque CYP1A2 is not biologically important. The main object of this study is elucidating the mode of natural selection on macaque CYP1A2.

\section{Gene conversion between CYP1A genes and effect on substrate specificity}

Because many factors, including gene conversion between paralogous genes, could create highly polymorphic genes, we first clarified the pattern of molecular evolution of the CYP1A subfamily. Whether CYP1A subfamily may have expanded or contracted many times through the process of birth-and-death evolution of gene families [32], is an interesting question. For example, in CYP2D subfamily, lineage-specific gene losses in primates were reported [33], but the subfamily also experienced a large-scale expansion in non-primate placental mammals. We found that many vertebrate species genomes encoded CYP1A1 and CYP1A2 with the same head-to-head orientation as the human genome, and macaques did not harbor any other paralogous CYP1A genes created by recent gene duplication. A new method combining a window plot and maximum likelihood successfully detected the signature of gene conversion at the first $500 \mathrm{bp}$ of exon 2 and entire exon 5, which correspond to SRS1 and SRS5, respectively. Our method is not sensitive to gene conversion tracts much shorter than the window size (150 bp), but successfully detected the gene conversion events in those regions. In addition, we did not find any signature of gene conversion using human and marmoset genes, indicating the last gene conversion event occurred before the divergence of Catarrhini and Platyrrhini. Because gene conversion is supposed to work effectively on young duplicates [34], we expect the two paralogs in primates continue diverging since gene conversion ceased. Interestingly, one amino acid at position 382 in SRS5 is responsible for substrate specificity of CYP1A1/2 gene to 7-ethoxyresorufin (382V) and 7-ethoxyresorufin (382L) [35]. The last gene conversion homogenized SRS5 of CYP1A1 and CYP1A2 and the substrate specificity should have been acquired after the event. Indeed, both macaque CYP1A1 and CYP1A2 proteins have $382 \mathrm{~V}$, but orangutan has $382 \mathrm{~V}$ in
CYP1A1 and 382L in CYP1A2, indicating that the mutation from valine to leucine that modified the substrate specificity fixed after the divergence of human and macaque lineages and before the divergence of human and orangutan lineages. In contrast to exon 2 and 5, the other exons showed much higher paralogous divergence. The regional heterogeneity of gene conversion pattern may indicate natural selection diversifying the substrate specificities of CYP1A1 and CYP1A2 has been maintaining the high paralogous divergence in those conversionrepressed regions, as shown in the cases of Drosophila transporter genes and yeast heat shock protein genes $[36,37]$.

\section{CYP1A2 gene is dispensable in macaques}

In contrast to our initial expectation, we found many null alleles were segregating both in $M$. fascicularis and M. mulatta CYP1A2. The highest allele frequency of these null alleles was 39\% at site 1066 in M. mulatta (Table 3). Although we previously identified two null alleles in macaque CYP3A4 and CYP $3 A 5$, the null allele frequencies were less than $1 \%$ [38], indicating the null variants in $C Y P 3 A 4 / 5$ are strongly selected against by natural selection in contrast to $C Y P 1 A 2$. Because all $M$. fascicularis so far examined had weak gene expression of CYP1A2, mutations in regulatory regions should have become fixed in the common ancestors of macaques. However, it remains unclear whether the initial loss of gene expression was driven by positive selection under the "less is more" hypothesis [39], or due to the fixation of neutral or slightly deleterious alleles.

\section{Mechanisms of rapid decay of CpG sites}

If macaque $C Y P 1 A 2$ gene is losing its function, why do we observe the high genetic diversity in populations? Although loss-of-function generally results in evolutionary patterns similar to patterns under neutrality, the genetic diversity in macaque $C Y P 1 A 2$ was significantly higher than $C Y P 1 A 1$, and much higher than the previously investigated background loci [13]. We found that primate CYP1A2 genes contained a considerable number of $\mathrm{CpG}$ sites, which are thought to mutate at much higher rates than other sites.

A number of mammalian genome studies have revealed that $\mathrm{CpG}$ sites are enriched in genic regions not only at nonsynonymous sites such as encoding arginine, but also at synonymous sites and introns. The reason why genic regions in mammalian genomes are enriched with CpG sites is not totally clear, but we could raise two hypotheses. One is the natural selection hypothesis, where natural selection is maintaining $\mathrm{CpG}$ sites within genes for the control of transcriptional regulation or integrity of genomes [40]. Another is the substitution bias hypothesis, where biased gene conversion 
is maintaining high GC content in genic regions [41] because of the association between recombination and gene density [42]. In particular, it was proposed that inter-locus gene conversion among multi-gene family members could increase CG content of those genes [43]. Although the latter mechanism would be important for creating the high GC regions in genomes, it cannot explain the deficiency of $\mathrm{CpG}$ degeneration in genic regions (Figure 3B), because under the substitution bias hypothesis we should observe many gain of CG as well as loss of CpG in genic regions. Therefore, at some extent, purifying selection protecting $\mathrm{CpG}$ sites within genic regions must play an essential role for maintaining $\mathrm{CpG}$ sites.

There are two ways how natural selection is protecting CpG sites from degeneration. Firstly, natural selection could keep CpG sites as unmethylated status in germ line cells, which can be observed in CpG-island associated genes. Secondly, CpG sites are methylated in germ line cells and tend to degenerate, but the degenerated alleles are removed from populations by purifying selection. Unfortunately, we could not determine which scenario is more plausible in the case of macaque CYP1A2 gene. Future studies investigating the methylation pattern within human and macaque CYP1A genes in many tissues would answer such questions. With either scenario, we could predict that once genes with many CpG sites lose its function, those genes would start to accumulate many mutations caused by rapid CPG degeneration.

\section{Effect of CpG site degeneration at species divergence level}

In this study, our main focus was the effect of $\mathrm{CpG}$ degeneration on genetic diversity within species. However, the same caution should be taken in analyses between two diverged species, as suggested by Subramanian and Kumar [44]. As an extreme case, imagine a gene encoding many arginine residues. If the gene lost its function and those sites are methylated, many CpG sites start to degenerate and we would observe the acceleration of nonsynonymous changes relative to synonymous changes. Such pattern is often interpreted as the signature of positive selection, but we need to be careful for interpreting such data [45-47]. Indeed, when we compare the coding sequences of human and $M$. fascicularis reference CYP1A2 sequences, the ratio of non-synonymous substitution rate to synonymous substitution rate was 0.445 , which was nearly two times higher than the genome-wide average [48], indicating that, although weakly, $\mathrm{CpG}$ degeneration affects the evolution rate of CYP1A2 at the apparent species divergence level.

\section{Conclusions}

Our molecular evolution and population genetic study strongly supports that macaque CYP1A2 is no longer essential for M. fascicularis and M. mulatta. At some time after the divergence between humans and macaques, the major hepatic CYP1A gene was changed in the lineage to macaques. However, CYP1A1 gene expression pattern in macaques has not been completely switched to the human $C Y P 1 A 2$ expression pattern; the expression of macaque CYP1A1 is not so abundant without induction, but strongly induced by omeprazole, a CYP inducer $[49,50]$. The alteration of gene expression pattern could be possible by changes in the gene regulatory region, because $C Y P 1 A 1$ and $C Y P 1 A 2$ are aligned in a head-to-head orientation and shared a promoter region [4]. At this moment, we do not have any hypothesis explaining why the change of expression pattern and loss of function occurred only in the macaque lineage. Since the last gene conversion events between $C Y P 1 A 1$ and CYP1A2 occurred before the divergence between Catarrhini and Platyrrhini, the resurrection of function by gene conversion cannot explain the macaque-specific gene loss. Because the functional importance of CYP1A1/2 genes is highly different between humans and macaques, we have to be cautious in extrapolating a drug-testing data using substrates metabolized by CYP1A genes from macaques to humans, despite of their somewhat overlapping substrate specificities.

\section{Methods}

\section{Sequence analysis}

DNA sequences of vertebrate $C Y P 1 A 1 / 2$ genes were retrieved from a public database, and analyzed using MEGA 5 [51]. DDBJ/EMBL/Genbank accession numbers of analyzed cDNA sequences are as follows: macaque CYP1A1 (D17575), macaque CYP1A2 (D86474), mouse CYP1A1 (AK005000), mouse CYP1A2 (BC018298), rat CYP1A1 (X00469), rat CYP1A2 (BC127476), chicken CYP1A4 (X99453), chicken CYP1A5 (X99454), and medaka CYP1A (AY297923). The nucleotide sequences were aligned using the MUSCLE with default parameter setting using the information of amino acid sequences [52]. The phylogenetic tree of CYP1A1/2 was constructed using the amino acid sequences with the Dayhoff's distance matrix and the bootstrap resampling was performed 1000 times.

\section{Test of gene conversion}

Quartet sequences (two paralogous genes from two different species) were used to detect the molecular evolution pattern under gene conversion. The nucleotide sequence alignments of exon 2, 3, and 7 were binned into 150 -bp-length windows with 9 bp incremental 
steps. Because the nucleotide sequence alignments of exon 4, 5, and 6 were shorter than $150 \mathrm{bp}$, they were analyzed as single windows. For each window, we estimated the log-likelihood values of the type- $\mathrm{N}$ and type$\mathrm{C}$ trees using PAML [22].

\section{Resequencing of macaque CYP1A1/2 genes}

Whole blood was collected from 63 cynomolgus macaques (31 from Indochina and 32 from Indonesia, 3-8 years of age, $3-5 \mathrm{~kg}$ ) and 28 rhesus macaques (from China, 2-3 years of age, weighing $3-5 \mathrm{~kg}$ ). Genomic DNA was prepared from these blood samples using Gentra Puregene Blood kit (Qiagen, Valencia, CA) according to the manufacturer's instructions. The study was reviewed and approved by the Institutional Animal Care and Use Committee of Shin Nippon Biomedical Laboratories, Ltd (Kainan, Japan). Polymorphisms were identified by PCR amplification, followed by directsequencing. Briefly, PCR was performed in a $20 \mu \mathrm{L}$ reaction containing $1 \mathrm{ng}$ of genomic DNA, 5 pmole of each primer, and 1 unit of AmpliTaq Gold DNA polymerase (Applied Biosystems, Foster City, CA) using a thermal cycler (Applied Biosystems). For exons 2-5 of CYP1A1, EX Taq HS DNA polymerase (Takara, Tokyo, Japan) was used. Thermal cycler condition included an initial denaturation at $95^{\circ} \mathrm{C}$ for $10 \mathrm{~min}$ : 30 cycles of $20 \mathrm{~s}$ at $95^{\circ}$ $\mathrm{C}, 30 \mathrm{~s}$ at $55-60^{\circ} \mathrm{C}$, and $1 \mathrm{~min}$ at $72^{\circ} \mathrm{C}$, followed by a final extension step of $5 \mathrm{~min}$ at $72^{\circ} \mathrm{C}$. PCR products were sequenced using an ABI PRISM BigDye Terminator v3.0 Ready Reaction Cycle Sequencing Kit (Applied Biosystems), followed by electrophoresis on an ABI PRISM 3730 DNA Analyzer (Applied Biosystems). PCR/ sequencing primers and annealing temperatures for PCR are listed in Table S1 (Additional File 1).

\section{Software Used}

DNA sequences of CYP1A1/2 genes in vertebrate species were analyzed using MEGA 5 [51]. Sequence data were analyzed using the Sequencher software (Gene Codes, Ann Arbor, MI). Polymorphism data were analyzed using DnaSP 5.0 [53].

\section{Additional material}

Additional file 1: Table S1. Primers used for PCR and sequencing.

\section{List of abbreviations}

CYP: cytochrome oxidase P450; SNP: single nucleotide polymorphism.

\section{Acknowledgements}

We would like to thank Dr. Hiroshi Akashi for comments and editing the manuscript and Mr. Akinori Matsushita for technical assistance. We wish to acknowledge valuable comments from three anonymous reviewers. This study was supported by Grant-in-Aid for Scientific Research on Innovative Areas (23113001 and 23113008) to N. O..

\section{Author details}

${ }^{1}$ Pharmacokinetics and Bioanalysis Center, Shin Nippon Biomedical Laboratories, Ltd., Kainan, Wakayama 642-0017, Japan. ²Department of Population Genetics, National Institute of Genetics, 1111 Yata, Mishima, Shizuoka 411-8540, Japan. ${ }^{3}$ Department of Genetics, The Graduate University for Advanced Studies (SOKENDAI), 1111, Yata, Mishima, Shizuoka 411-8540, Japan.

\section{Authors' contributions}

$\mathrm{NO}$ and YU designed the research. YU carried out the experiments. NO and YU analyzed the data and wrote the paper. Both authors have read and approved the final manuscript.

Received: 2 August 2011 Accepted: 1 October 2011

Published: 1 October 2011

\section{References}

1. Anzenbacher P, Anzenbacherová E: Cytochromes P450 and metabolism of xenobiotics. Cell Mol Life Sci 2001, 58(5):737-747.

2. Dearing MD, Foley WJ, Mclean S: The influence of plant secondary metabolites on the nutritional ecology of herbivorous terrestrial vertebrates. Annu Rev Ecol Evol Syst 2005, 36:169-189.

3. Nelson DR, Zeldin DC, Hoffman SM, Maltais LJ, Wain HM, Nebert DW: Comparison of cytochrome P450 (CYP) genes from the mouse and human genomes, including nomenclature recommendations for genes, pseudogenes and alternative-splice variants. Pharmacogenet Genomics 2004, 14(1):1-18.

4. Corchero J, Pimprale S, Kimura S, Gonzalez FJ: Organization of the CYP1A cluster on human chromosome 15: Implications for gene regulation. Pharmacogenet Genomics 2001, 11(1):1-6.

5. Morrison HG, Weil EJ, Karchner Sl, Sogin ML, Stegeman JJ: Molecular cloning of CYP1A from the estuarine fish fundulus heteroclitus and phylogenetic analysis of CYP1 genes: Update with new sequences. Comp Biochem Phys C 1998, 121(1-3):231-240.

6. Omiecinski CJ, Remmel RP, Hosagrahara VP: Concise review of the cytochrome P450s and their roles in toxicology. Toxicol Sci 1999, 48(2):151-156

7. Amos Cl, Caporaso NE, Weston A: Host factors in lung cancer risk: A review of interdisciplinary studies. Cancer Epidem Biomar 1992, 1(6):505-513.

8. Browning SL, Tarekegn A, Bekele E, Bradman N, Thomas MG: CYP1A2 is more variable than previously thought: $A$ genomic biography of the gene behind the human drug-metabolizing enzyme. Pharmacogenet Genomics 2010, 20(11):647-664.

9. Jorge-Nebert LF, Jiang Z, Chakraborty R, Watson J, Jin L, Mcgarvey ST, Deka R, Nebert DW: Analysis of human CYP1A1 and CYP1A2 genes and their shared bidirectional promoter in eight world populations. Hum Mutat 2010, 31(1):27-40.

10. Uno $Y$, Iwasaki $K$, Yamazaki H, Nelson DR: Macaque cytochromes P450: Nomenclature, transcript, gene, genomic structure, and function. Drug Metab Rev 2011, 1-16.

11. Suntharalingam G, Perry MR, Ward S, Brett SJ, Castello-Cortes A, Brunner MD, Panoskaltsis N: Cytokine storm in a phase 1 trial of the antiCD28 monoclonal antibody TGN1412. N Engl J Med 2006, 355(10):1018-1028.

12. Edwards RJ, Murray BP, Murray S, Schulz T, Neubert D, Gant TW, Thorgeirsson SS, Boobis AR, Davies DS: Contribution of CYP1A1 and CYP1A2 to the activation of heterocyclic amines in monkeys and human. Carcinogenesis 1994, 15(5):829-836.

13. Osada N, Uno Y, Mineta K, Kameoka Y, Takahashi I, Terao K: Ancient genome-wide admixture extends beyond the current hybrid zone between Macaca fascicularis and M. mulatta. Mol Ecol 2010, 19(14):2884-2895.

14. Levene $\mathrm{H}$ : Genetic equilibrium when more than one ecological niche is available. Am Nat 1953, 87(836):331-333.

15. Charlesworth D: Balancing selection and its effects on sequences in nearby genome regions. PLOS Genet 2006, 2(4):e64. 
16. Sakuma T, Hieda M, Igarashi T, Ohgiya S, Nagata R, Nemoto N, Kamataki T: Molecular cloning and functional analysis of cynomolgus monkey CYP1A2. Biochem Pharmacol 1998, 56(1):131-139.

17. Uno Y, Uehara S, Murayama N, Yamazaki H: CYP1D1, pseudogenized in human, is expressed and encodes a functional drug-metabolizing enzyme in cynomolgus monkey. Biochem Pharmacol 2011, 81(3):442-450

18. Goldstone $\mathrm{H}$, Stegeman J: A revised evolutionary history of the CYP1A subfamily: Gene duplication, gene conversion, and positive selection. J Mol Evol 2006, 62(6):708-717.

19. Benton MJ: Phylogeny of the major tetrapod groups: Morphological data and divergence dates. J Mol Evol 1990, 30(5):409-424.

20. Kumar S, Hedges SB: A molecular timescale for vertebrate evolution Nature 1998, 392(6679):917-920.

21. Uno $Y$, Suzuki $Y$, Wakaguri H, Sakamoto $Y$, Sano H, Osada N, Hashimoto K, Sugano S, Inoue I: Expressed sequence tags from cynomolgus monkey (Macaca fascicularis) liver: A systematic identification of drugmetabolizing enzymes. FEBS Lett 2008, 582(2):351-358

22. Yang Z: PAML 4: Phylogenetic analysis by maximum likelihood. $\mathrm{Mol}$ Biol Evol 2007, 24(8):1586-1591.

23. Hudson RR, Kreitman M, Aguade M: A test of neutral molecular evolution based on nucleotide data. Genetics 1987, 116(1):153-159.

24. Elango N, Kim S-H, Vigoda E, Yi SV, Program NCS: Mutations of different molecular origins exhibit contrasting patterns of regional substitution rate variation. PLoS Comput Biol 2008, 4(2):e1000015.

25. Roach JC, Glusman G, Smit AFA, Huff CD, Hubley R, Shannon PT, Rowen L, Pant KP, Goodman N, Bamshad M, et al: Analysis of genetic inheritance in a family quartet by whole-genome sequencing. Science 2010 328(5978):636-639.

26. Maglott D, Ostell J, Pruitt KD, Tatusova T: Entrez Gene: Gene-centered information at NCBI. Nucleic Acids Res 2011, 39(suppl 1):D52-D57.

27. Bernardi G: Isochores and the evolutionary genomics of vertebrates. Gene 2000, 241(1):3-17.

28. Naumann C, Hartmann T, Ober D: Evolutionary recruitment of a flavindependent monooxygenase for the detoxification of host plant-acquired pyrrolizidine alkaloids in the alkaloid-defended arctiid moth Tyria jacobaeae. Proc Natl Acad Sci USA 2002, 99(9):6085-6090.

29. Li X, Baudry J, Berenbaum MR, Schuler MA: Structural and functional divergence of insect CYP6B proteins: From specialist to generalist cytochrome P450. Proc Natl Acad Sci USA 2004, 101(9):2939-2944.

30. Thompson EE, Kuttab-Boulos H, Witonsky D, Yang L, Roe BA, Di Rienzo A: CYP3A variation and the evolution of salt-sensitivity variants. Am J Hum Genet 2004, 75(6):1059-1069.

31. Voight BF, Kudaravalli $S$, Wen $X$, Pritchard JK: A map of recent positive selection in the human genome. PLOS Biol 2006, 4(3):e72.

32. Nei M, Rooney AP: Concerted and birth-and-death evolution of multigene families. Annu Rev Genet 2005, 39:121-152.

33. Yasukochi $Y$, Satta $Y$ : Evolution of the CYP2D gene cluster in humans and four non-human primates. Genes Genet Syst 2011, 86(2):109-116.

34. Teshima KM, Innan $\mathrm{H}$ : The effect of gene conversion on the divergence between duplicated genes. Genetics 2004, 166(3):1553-1560.

35. Liu J, Ericksen SS, Sivaneri M, Besspiata D, Fisher CW, Szklarz GD: The effect of reciprocal active site mutations in human cytochromes P450 1A1 and 1 A2 on alkoxyresorufin metabolism. Arch Biochem Biophys 2004, 424(1):33-43.

36. Osada N, Innan H: Duplication and gene conversion in the drosophila melanogaster genome. PLoS Genet 2008, 4(12):e1000305.

37. Takuno $\mathrm{S}$, Innan $\mathrm{H}$ : Selection to maintain paralogous amino acid differences under the pressure of gene conversion in the heat-shock protein genes in yeast. Mol Biol Evol 2009, 26(12):2655-2659.

38. Uno Y, Matsushita A, Osada N, Uehara S, Kohara S, Nagata R, Fukuzaki K, Utoh M, Murayamay N, Yamazaki H: Genetic variants of CYP3A4 and CYP3A5 in cynomolgus and rhesus macaques. Drug Metab Dispos 2010, 38(2):209-214

39. Olson MV: When less is more: Gene loss as an engine of evolutionary change. The Am J Hum Genet 1999, 64(1):18-23.

40. Cross SH, Bird AP: CpG islands and genes. Curr Opin Genet Dev 1995, 5(3):309-314

41. Galtier N, Duret L: Adaptation or biased gene conversion? Extending the null hypothesis of molecular evolution. Trends Genet 2007, 23(6):273-277.

42. Kong A, Gudbjartsson DF, Sainz J, Jonsdottir GM, Gudjonsson SA, Richardsson B, Sigurdardottir S, Barnard J, Hallbeck B, Masson G, et al: A high-resolution recombination map of the human genome. Nat Genet 2002, 31(3):241-247.

43. Galtier N: Gene conversion drives gc content evolution in mammalian histones. Trends Genet 2003, 19(2):65-68.

44. Subramanian S, Kumar S: Neutral substitutions occur at a faster rate in exons than in noncoding DNA in primate genomes. Genome Res 2003, 13(5):838-844.

45. Hughes AL: Looking for Darwin in all the wrong places: The misguided quest for positive selection at the nucleotide sequence level. Heredity 2007, 99(4):364-373.

46. Subramanian S, Kumar S: Higher intensity of purifying selection on $>90 \%$ of the human genes revealed by the intrinsic replacement mutation rates. Mol Biol Evol 2006, 23(12):2283-2287.

47. Suzuki Y, Gojobori T, Kumar S: Methods for incorporating the hypermutability of $\mathrm{CpG}$ dinucleotides in detecting natural selection operating at the amino acid sequence level. Mol Biol Evol 2009, 26(10):2275-2284.

48. Wang HY, Chien HC, Osada N, Hashimoto K, Sugano S, Gojobori T, Chou CK, Tsai SF, Wu Cl, Shen CK: Rate of evolution in brain-expressed genes in humans and other primates. PLOS Biol 2007, 5:e13.

49. Nishimura M, Koeda A, Suganuma Y, Suzuki E, Shimizu T, Nakayama M, Satoh T, Narimatsu S, Naito $S$ : Comparison of inducibility of CYP1A and CYP3A mRNAs by prototypical inducers in primary cultures of human cynomolgus monkey, and rat hepatocytes. Drug Metab Pharmacokinet 2007, 22(3):178-186.

50. Ise R, Uehara S, Akiyama H, Kondo S, Iwasaki K, Nagata R, Nobumasa H, Yamazaki $\mathrm{H}$, Uno Y: A newly developed DNA microarray is useful to assess induction of cytochromes P450 in cynomolgus monkey. Drug Metab Pharmacokinet 2011, 26(3):228-235.

51. Tamura K, Peterson D, Peterson N, Stecher G, Nei M, Kumar S: MEGA5: Molecular evolutionary genetics analysis using maximum likelihood, evolutionary distance, and maximum parsimony methods. Mol Biol Evol 2011, 28(10):2731-2739.

52. Edgar RC: MUSCLE: Multiple sequence alignment with high accuracy and high throughput. Nucleic Acids Res 2004, 32(5):1792-1797.

53. Librado P, Rozas J: DnaSP v5: A software for comprehensive analysis of DNA polymorphism data. Bioinformatics 2009, 25(11):1451-1452.

doi:10.1186/1471-2148-11-283

Cite this article as: Uno and Osada: CpG site degeneration triggered by the loss of functional constraint created a highly polymorphic macaque drug-metabolizing gene, CYP1A2. BMC Evolutionary Biology 2011 11:283.

\section{Submit your next manuscript to BioMed Central and take full advantage of:}

- Convenient online submission

- Thorough peer review

- No space constraints or color figure charges

- Immediate publication on acceptance

- Inclusion in PubMed, CAS, Scopus and Google Scholar

- Research which is freely available for redistribution

Submit your manuscript a www.biomedcentral.com/submit
C Biomed Central 